\title{
Oxidative stress and gut-derived lipopolysaccharides in children affected by paediatric autoimmune neuropsychiatric disorders associated with streptococcal infections
}

Lorenzo Loffredo ${ }^{1 *+}$, Alberto Spalice ${ }^{2 \dagger}$, Francesca Salvatori ${ }^{2}$, Giovanna De Castro ${ }^{2}$, Cristiana Alessia Guido ${ }^{2}$, Anna Maria Zicari ${ }^{2}$, Paolo Ciacci ${ }^{1}$, Simona Battaglia ${ }^{1}$, Giulia Brindisi ${ }^{2}$, Evaristo Ettorre ${ }^{1}$, Cristina Nocella ${ }^{3,4}$, Guglielmo Salvatori ${ }^{5}$, Marzia Duse ${ }^{2}$, Francesco Violi ${ }^{1,4}$ and Roberto Carnevale ${ }^{3,4}$

\begin{abstract}
Background: Paediatric autoimmune neuropsychiatric disorders associated with streptococcal infections syndrome (PANDAS) identifies patients with acute onset of obsessive-compulsive and tic disorders. The objective of this study was to evaluate serum NOX2 levels, as well as 8-iso-prostaglandin F2a (8-iso-PGF2a) and lipopolysaccharide (LPS) of PANDAS patients.

Methods: In this study we wanted to compare serum levels of soluble NOX2-dp (sNOX-2-dp), iso-PGF2a and LPS in 60 consecutive subjects, including 30 children affected by PANDAS and 30 controls (CT) matched for age and gender. Serum zonulin was used as intestinal permeability assay.

Results: Compared with CT, PANDAS children had increased serum levels of sNOX-2-dp, 8-iso-PGF2a and LPS. Bivariate analysis showed that serum sNOX2-dp was significantly correlated with LPS ( $R s=0.359 ; p=0.005$ ), zonulin (Rs $=0.444 ; p<0.001)$ and 8-iso-PGF2a (Rs $=0.704 ; p<0.001$ ). Serum LPS significantly correlated with zonulin (Rs $=$ $0.610 ; p<0.001$ ), and 8-iso-PGF2a (Rs $=0.591 ; p=0.001$ ). Finally, a multiple linear regression analysis showed that serum 8-iso-PGF2 $a$ and zonulin were the only independent variables associated with sNOX2-dp $\left(R^{2}=68 \%\right)$.
\end{abstract}

Conclusion: This study shows that children affected by PANDAS have high circulating levels of sNOX2-dp, isoprostanes and of LPS that could be involved in the process of neuroinflammation.

Keywords: PANS, PANDAS, NOX2, NADPH oxidase, Oxidative stress, LPS

\footnotetext{
* Correspondence: lorenzo.loffredo@uniroma1.it

${ }^{\dagger}$ Lorenzo Loffredo and Alberto Spalice are co-first authors

'Department of Clinical, Internal, Anaesthetic and Cardiovascular Sciences,

Sapienza University of Rome, I Clinica Medica, Viale del Policlinico 155, 00161 Rome, Italy

Full list of author information is available at the end of the article
}

(C) The Author(s). 2020 Open Access This article is licensed under a Creative Commons Attribution 4.0 International License, which permits use, sharing, adaptation, distribution and reproduction in any medium or format, as long as you give appropriate credit to the original author(s) and the source, provide a link to the Creative Commons licence, and indicate if changes were made. The images or other third party material in this article are included in the article's Creative Commons licence, unless indicated otherwise in a credit line to the material. If material is not included in the article's Creative Commons licence and your intended use is not permitted by statutory regulation or exceeds the permitted use, you will need to obtain permission directly from the copyright holder. To view a copy of this licence, visit http://creativecommons.org/licenses/by/4.0/ The Creative Commons Public Domain Dedication waiver (http://creativecommons.org/publicdomain/zero/1.0/) applies to the data made available in this article, unless otherwise stated in a credit line to the data. 


\section{Background}

Paediatric acute-onset neuropsychiatric syndrome (PANS) is defined as a wide spectrum of disorders characterised by sudden onset of obsessive-compulsive disorder (OCD) or severely restricted food intake in children [1-3]. A particular subtype of PANS is considered the paediatric autoimmune neuropsychiatric disorders associated with streptococcal infections syndrome (PANDAS) that identifies patients with acute onset of obsessive-compulsive and/or tic disorders related to Group-A streptococcus (GAS) infection [2, 4]. The close relation with streptococcus infection led to hypothesize to an autoimmune pathogenesis of PANDAS in which streptococcal antibodies cross-react with neuronal antigens [5]. This latter elicits dysregulation of dopamine receptors placed in the basal ganglia and in other types of neurons in the cortex [6] and to a persisting neuroinflammation [7].

Growing evidence showed that oxidative stress has an important role in the neuroinflammation process as shown in neurodegenerative disease and in psychotic disorders [8, 9]. Previous experimental studies found that reactive oxygen species (ROS), derived from NADPH oxidase-2 (NOX2), have a pivotal role in apoptotic pathways and in mediating the inflammatory processes in the central nervous system $[10,11]$. To the best of our knowledge, no study has analyzed oxidative stress and NADPH oxidase activation in children affected by PANDAS.

Several studies in animals and humans suggested that alterations of gut microbiota are associated to neuroinflammation [12, 13]. A recent study by Quagliarello et al. showed that children affected by PANDAS have changes of the gut microbiota that could favor the neuro-inflammation [14].

Lipopolysaccharide (LPS), derived from gram-negative bacteria, plays a pivotal role in causing neuroinflammation by an increase of oxidative stress $[12,15,16]$. A relationship among LPS, oxidative stress and NOX2 activation, in other clinical situations as NAFLD [17], pneumonia [18] atherosclerosis [19] and neurodegenerative disease [16], has been previously reported. We speculated that children affected by PANDAS have NOX2 over-activation and increased oxidative stress that may favor to onset and persistence of the disease. Thus, this study wanted to assess NOX2 and 8-iso-prostaglandin F2 $\alpha$ (8-iso-PGF $2 \alpha$ ), as markers of oxidative stress, in serum of PANDAS and controls. Furthermore, we wanted to assess the potential role for gut-derived LPS in eliciting systemic NOX2 levels in children affected by PANDAS.

\section{Methods}

Thirty consecutive subjects (24 males and 6 females, mean age $9 \pm 3$ ), who were referred to the Allergology and Pediatric Neurology clinic of "Sapienza" University of Rome from January 2018 to December 2019, were enrolled. Thirty control subjects (24 males and 6 females, mean age $9 \pm 3$ ), matched for aged and gender, were enrolled at the same pediatric clinic at the same period. Controls were recruited through a screening program in childhood.

Inclusion criteria were represented by: subjects aged between 3 and 16 years affected by PANDAS.

PANDAS was defined according to the criteria elaborated by Dr. Swedo and collaborators [2, 3]:

1) presence of OCD (diagnosed according to DSM IV criteria) and/or tic disorders.

2) onset of symptoms between 3 years and puberty.

3) episodic course of the disease.

4) symptoms and exacerbations temporally associated with GAS infections.

5) association with neurological anomalies (choreiform movements and motor hyperactivity during symptoms exacerbations).

Exclusion criteria were represented by: PANS not related to GAS, Sydenham corea, Tourette syndrome, Autoimmune encephalitis, Systemic autoimmune diseases, Wilson's disease, congenital heart disease, renal disease, cancer, treatment with immuno-suppressive drugs or antioxidants, liver disease, acute disease.

The study conformed to the ethical guidelines of the 1975 Declaration of Helsinki and was approved by the Sapienza University of Rome Ethics Committee (n. 5377).

\section{Blood sampling}

Blood samples were collected, between 8.00 and $9.00 \mathrm{am}$, in Vacutainers (Vacutainer Systems, Belliver Industrial Estate, Plymouth, UK) after an overnight fast (12 h). Samples were centrifuged at $300 \mathrm{~g}$ for $10 \mathrm{~min}$, and the supernatant was collected and stored at $-80^{\circ} \mathrm{C}$ until dosage.

\section{ELISA detection of sNOX2-dp}

Serum NOX2 levels were measured as soluble NOX2derived peptide (sNOX2-dp) with an ELISA method as previously reported [20]. Briefly, reference standards of sNOX2-dp and serum samples were prepared and the wells of a microtiter plate were coated overnight at $4{ }^{\circ} \mathrm{C}$. After washing and blocking the remaining proteinbinding sites, a specific anti-sNOX2dp-horseradish peroxidase (HRP) monoclonal antibody against the amino acidic sequence of the extra membrane portion of NOX2 was added in each well. Finally, after the addition of the substrate 3,3',5,5'-tetramethylbenzidine (TMB, Bethyl Laboratories, TX, USA) and the stop solution, the 
absorbance of each well was read spectrophotometrically at $450 \mathrm{~nm}$ with a plate reader. Values were expressed as $\mathrm{pg} / \mathrm{ml}$; intra-assay and inter-assay coefficients of variation were 8.95 and $9.01 \%$, respectively.

\section{8-iso-PGF2a}

8 -iso-PGF2 $\alpha$ levels were measured in serum by using a colorimetric assay kit (DRG International, Inc). Values were expressed as $\mathrm{pmol} / \mathrm{L}$. Intra-assay and inter-assay coefficients of variation were 5.8 and $5.0 \%$, respectively.

\section{Serum zonulin}

Serum zonulin levels were measured with a commercially ELISA kit (Elabscience). Briefly, standards and samples were added to a pre-coated microplate with a specific antibody for zonulin and incubated $90 \mathrm{~min}$ at $37^{\circ} \mathrm{C}$. Then, a biotinylated detection antibody and Avidin-Horseradish Peroxidase (HRP) conjugate were added to each well. The amount of zonulin was measured at a wavelength of $450 \mathrm{~nm}$ with a microplate autoreader. Values were expressed as $\mathrm{ng} / \mathrm{ml}$; both intra-assay and inter-assay coefficients of variation were within $10 \%$.

\section{LPS}

Samples were thawed only once and used to perform specific sandwich enzyme-linked immunosorbent assay (ELISA) to measure LPS (Cusabio, Wuhan, China). The standards and samples were plated into a micro-plate pre-coated with the antibody specific for LPS. After incubation, samples were read at $450 \mathrm{~nm}$. Values were expressed as $\mathrm{pg} / \mathrm{ml}$; intra-assay and inter-assay coefficients of variation were $<10 \%$.

\section{Statistical analysis}

Statistical analysis was performed with SPSS 18.0 software for Windows (SPSS, Chicago, IL, USA). The Kolmogorov-Smirnov test was used to determine whether variables were normally distributed. Normally distributed data are described as means \pm standard deviations (SDs). Group differences were analyzed by Kruskal-Wallis tests (for non-normally distributed data) or analysis of variance (ANOVA). Differences between categorical variables were assessed by the $\chi^{2}$ test. Simple linear regression analysis was performed by Spearman's rank correlation test; the variables with evidence of an association $p<0.10$ were included in a multivariable linear regression using an automated procedure. A $p$ value $<0.05$ was considered as statistically significant.

\section{Sample size determination}

The minimum sample size was computed with respect to a two-tailed, one-sample Student $t$ test considering, on the basis of data from a previous pilot study (data not shown): a difference of $4 \mathrm{pg} / \mathrm{ml}$ for sNOX2dp levels between children affected by PANDAS and controls, 4.7 as $\mathrm{SD}, 0.05(\alpha)$ as type I error probability and 0.95 as power $1-\beta$. The sample size was $n=30$ patients/group.

\section{Results}

Clinical characteristics of patients with PANDAS and controls are showed in the Table 1. No significant difference between PANDAS and controls was found for age, fasting blood glucose, blood pressure and BMI (Table 1). Conversely serum sNOX2-dp, 8-iso-PGF2 $\alpha$, LPS and zonulin were higher in PANDAS compared to controls (Table 1 and Fig. 1, panels a-d).

Bivariate analysis showed that serum sNOX2-dp was significantly correlated with 8 -iso-PGF $2 \alpha \quad(\mathrm{Rs}=0.704$; $p<0.001$ ), LPS (Rs $=0.359 ; p=0.005)$ and zonulin (Rs $=$ $0.444 ; p<0.001)$. The same statistical analysis showed that serum LPS significantly correlated with 8-isoPGF $2 \alpha(R s=0.591 ; p<0.001)$ and zonulin $(\mathrm{Rs}=0.610$; $p<0.001)$. Furthermore, serum 8-iso-PGF $2 \alpha$ correlated with the tic disorders ( $\mathrm{Rs}=0.382 ; p=0.03$ ).

Multiple linear regression analyses, showed that serum 8-iso-PGF2 $\alpha$ (SE: 0.011; standardized coefficient $\beta$ : $0.780 ; p<0.001$ ) and zonulin (SE: 0.911; standardized coefficient $\beta$ : $0.241 ; P=0.04$ ) were the only independent predictive variables associated with $\operatorname{sNOX2-dp}\left(R^{2}=\right.$ $68 \%)$.

\section{Discussion}

This study reports that patients with PANDAS have high sNOX-2-dp levels and suggests a potential role for gut microbiota as a source of oxidative stress in this population.

NOX-2 derived oxidative stress leads to inflammation in several neurologic diseases as Amyotrophic lateral sclerosis, Parkinson's disease and Alzheimer's disease $[10,16]$. Furthermore, NOX2 activation seems to be involved in the pathogenesis of psychotic disorders, as schizophrenia, leading to an imbalance of excitation and inhibition in cortical neural circuits [21]. To the best of our knowledge, NOX2 activation and oxidative stress has never been studied in patients with PANS and PANDAS. This study reports that PANDAS subjects have high levels of sNOX2-dp and high levels of isoprostanes, suggesting an increased systemic oxidative stress derived from NOX2 activation in this neuropsychiatric disorder. Another interesting result of this study is the positive correlation between TIC and isoprostanes, hypothesizing a direct relationship between oxidative stress and neuropsychiatric manifestation in PANDAS.

Previous studies identified dysbiosis in patients suffering from neurologic diseases and proposed the concept of "gut-brain-axis" as source of neuroinflammation [2224]. Recently, Quagliarello et al. showed that children affected by PANDAS also have gut dysbiosis [14]. 
Table 1 Clinical and laboratory characteristics of PANDAS and controls

\begin{tabular}{llll}
\hline & PANDAS $(\boldsymbol{n}=30)$ & Controls $(\boldsymbol{n}=30)$ & $\boldsymbol{p}$ value \\
\hline Age & $9 \pm 3$ & $9 \pm 3$ & 0.949 \\
Gender (male/female) & $24 / 6$ & $24 / 6$ & 1 \\
Glycaemia (mg/dL) & $83 \pm 3.75$ & $87 \pm 3$ & 0.617 \\
Systolic blood pressure $(\mathbf{m m H g})$ & $101 \pm 3.79$ & $112 \pm 3$ & 0.198 \\
Diastolic blood pressure $(\mathbf{m m H g})$ & $67 \pm 2.55$ & $70 \pm 2$ & 0.6 \\
BMI & $18 \pm 2$ & $17 \pm 1$ & 0.157 \\
Tic disorders (presence/absence) & $25 / 5$ & 0 & - \\
OCD (presence/ absence) & $10 / 20$ & 0 & - \\
Anti-streptolisinic title (Ul/mL) & $409 \pm 262$ & 0 & $<0.001$ \\
LPS (pg/ml) & $24.1 \pm 9.2$ & $8.1 \pm 3.6$ & 0.02 \\
sNOX2-dp (pg/ml) & $20.4 \pm 8.1$ & $16.3 \pm 5.7$ & 0.005 \\
Zonulin (ng/ml) & $2.6 \pm 1$ & $1.7 \pm 0.6$ & 0.001 \\
8-iso-PGF2a & $175 \pm 84$ & $106 \pm 43$ &
\end{tabular}

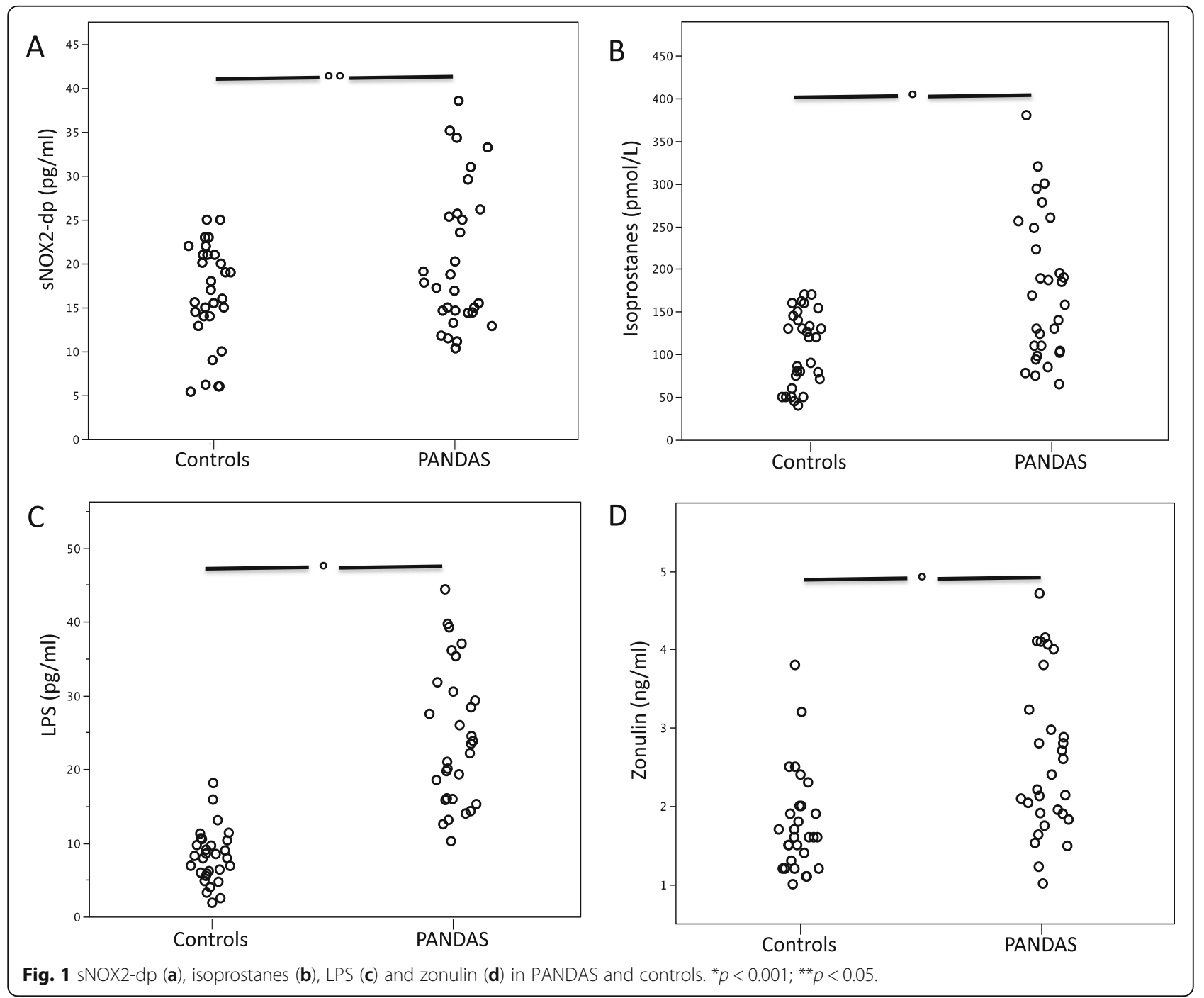


Furthermore, the same authors hypothesized that streptococcal infections alter gut microbiota and consequently lead to a proinflammatory state in the gut by selection of specific bacterial strains [14]. Gram negative bacteria of gastro-intestinal tract secrete LPS that exerts proinflammatory actions on neurons [15]. Animal studies showed that LPS increases neuroinflammation by NOX2 activation $[15,25,26]$; however, the mechanism through which LPS damages the nervous system is unclear.

LPS has been hypothesized to have a pathogenetic role in PANDAS [27], although no study evaluated LPS serum levels in this neuropsychiatric disorder. Thus, to address this issue we studied LPS levels in PANDAS. We found that subjects affected by PANDAS disease have higher LPS levels that are linearly associated with sNOX2-dp levels and with isoprostanes. This association suggests a link between LPS and oxidative stress in PANDAS. However, further studies are needed to establish the pathophysiological mechanisms responsible for neuroinflammatory process.

To evaluate whether gut permeability can explain the LPS increase in PANDAS, we assessed the circulating levels of zonulin, which modulate gut permeability by disassembling the intercellular tight junctions [28]. Previous studies showed that zonulin up-regulation increases gut permeability [29]. The high serum levels of zonulin in PANDAS patients and its correlation with serum LPS provide the evidence that gut permeability is enhanced in this neuropsychiatric disorder and may be responsible for the increased circulating levels of LPS.

The study has some limitations. NOX2 and oxidative stress were studied in blood and not in the nervous system by biopsies. However, this procedure is unethical and invasive. Furthermore, we did not assess other NADPH isoforms, such as NOX1 and NOX4, which could also increase oxidative stress. The present study did not address the mechanism that contribute to the translocation of LPS from the gut microbiota to the central nervous system. However, changes in intestinal permeability may be a plausible mechanism, since an increase in serum zonulin is significantly correlated with blood LPS.

\section{Conclusions}

This study shows that children affected by PANDAS have high circulating levels of sNOX2-dp, isoprostanes and of LPS that could be potentially implicated in the process of neuroinflammation.

\footnotetext{
Abbreviations

PANS: Pediatric acute-onset neuropsychiatric syndrome; PANDAS: Pediatric autoimmune neuropsychiatric disorders associated with streptococcal infections syndrome; NADPH oxidase: Nicotinamide-adenine dinucleotide phosphate oxidase; 8-iso-PGF2a: 8-iso-prostaglandin F2a;

LPS: Lipopolysaccharide; OCD: Obsessive-compulsive disorder; GAS: Group-A streptococcus
}

Acknowledgements

Thank you to Veronica Angelici MD for her support in reviewing the article.

\section{Authors' contributions}

LL and AS conceptualized and designed the study, drafted the initial manuscript, reviewed and revised the manuscript. FS, PC, SB, GB, GS, EE, CG, AZ and GD collected data, carried out the initial analyses, and reviewed and revised the manuscript. CN performed laboratory analyses, reviewed and revised the manuscript. FV, RC and MD conceptualized and designed the study, coordinated and supervised data collection, and critically reviewed the manuscript for important intellectual content. All authors approved the final manuscript as submitted and agree to be accountable for all aspects of the work.

\section{Funding}

This study was supported by a grant from the University of Rome "La Sapienza" (Università degli Studi di ROMA "la Sapienza" Progetti di Ateneo 2018 n.: RM1 1816427485472 - funds to L.L.). The funders had no role in the study design or collection, analysis, or interpretation of data, nor in writing this manuscript.

\section{Availability of data and materials}

The data used to support the findings of this study are available from the corresponding author upon request.

\section{Ethics approval and consent to participate}

The study was approved by the Rome Ethics Committee of Sapienza University (n. 5377).

Written informed consent for participation in the study was obtained for subjects under 16 years old from their parent or guardian.

\section{Consent for publication}

Not applicable.

\section{Competing interests}

The authors declare that they have no competing interests.

\section{Author details}

'Department of Clinical, Internal, Anaesthetic and Cardiovascular Sciences, Sapienza University of Rome, I Clinica Medica, Viale del Policlinico 155, 00161 Rome, Italy. ${ }^{2}$ Department of Pediatrics, Sapienza University of Rome, Rome 00161, Italy. ${ }^{3}$ Department of Medical-Surgical Sciences and Biotechnologies, Sapienza University of Rome, Latina, Italy. ${ }^{4}$ Mediterranea Cardiocentro, Naples, Italy. ${ }^{5}$ Neonatal Intensive Care Unit, Bambino Gesù Pediatric Hospital, Rome, Italy.

Received: 12 December 2019 Accepted: 11 March 2020

Published online: 18 March 2020

\section{References}

1. Hesselmark E, Bejerot S. Biomarkers for diagnosis of pediatric acute neuropsychiatric syndrome (PANS) - sensitivity and specificity of the Cunningham panel. J Neuroimmunol. 2017;312:31-7.

2. Swedo SE, Leonard HL, Garvey M, Mittleman B, Allen AJ, Perlmutter S, Lougee L, Dow S, Zamkoff J, Dubbert BK. Pediatric autoimmune neuropsychiatric disorders associated with streptococcal infections: clinical description of the first 50 cases. Am J Psychiatry. 1998;155(2):264-71.

3. Swedo SL, Rose N. From research subgroup to clinical syndrome: modifying the PANDAS criteria to describe PANS (pediatric acute-onset neuropsychiatric syndrome). Pediatr Therapeut. 2012;2(2):1-8.

4. Murciano M, Biancone DM, Capata G, Tristano I, Martucci V, Guido CA, Anaclerio S, Loffredo L, Zicari AM, Duse M, et al. Focus on cardiologic findings in 30 children with PANS/PANDAS: an Italian single-center observational study. Front Pediatr. 2019;7:395.

5. Murphy TK, Kurlan R, Leckman J. The immunobiology of Tourette's disorder, pediatric autoimmune neuropsychiatric disorders associated with Streptococcus, and related disorders: a way forward. J Child Adolesc Psychopharmacol. 2010;20(4):317-31.

6. Cunningham MW, Cox CJ. Autoimmunity against dopamine receptors in neuropsychiatric and movement disorders: a review of Sydenham chorea and beyond. Acta Physiol (Oxford). 2016;216(1):90-100. 
7. Kumar A, Williams MT, Chugani HT. Evaluation of basal ganglia and thalamic inflammation in children with pediatric autoimmune neuropsychiatric disorders associated with streptococcal infection and tourette syndrome: a positron emission tomographic (PET) study using 11C-[R]-PK11195. J Child Neurol. 2015;30(6):749-56.

8. Niedzielska E, Smaga I, Gawlik M, Moniczewski A, Stankowicz P, Pera J, Filip M. Oxidative stress in neurodegenerative diseases. Mol Neurobiol. 2016; 53(6):4094-125.

9. Barron H, Hafizi S, Andreazza AC, Mizrahi R. Neuroinflammation and Oxidative Stress in Psychosis and Psychosis Risk. Int J Mol Sci. 2017;18(3):651.

10. Sorce $\mathrm{S}$, Krause KH. NOX enzymes in the central nervous system: from signaling to disease. Antioxid Redox Signal. 2009;11(10):2481-504.

11. Cahill-Smith S, Li JM. Oxidative stress, redox signalling and endothelial dysfunction in ageing-related neurodegenerative diseases: a role of NADPH oxidase 2. Br J Clin Pharmacol. 2014;78(3):441-53.

12. Quigley EMM. Microbiota-brain-gut Axis and neurodegenerative diseases. Curr Neurol Neurosci Rep. 2017;17(12):94.

13. Clapp M, Aurora N, Herrera L, Bhatia M, Wilen E, Wakefield S. Gut microbiota's effect on mental health: the gut-brain axis. Clin Pract. 2017;7(4): 987.

14. Quagliariello A, Del Chierico F, Russo A, Reddel S, Conte G, Lopetuso LR, laniro G, Dallapiccola B, Cardona F, Gasbarrini A, et al. Gut microbiota profiling and gut-brain crosstalk in children affected by pediatric acuteonset neuropsychiatric syndrome and pediatric autoimmune neuropsychiatric disorders associated with streptococcal infections. Front Microbiol. 2018;9:675.

15. Zhao Y, Jaber V, Lukiw WJ. Secretory products of the human Gl tract microbiome and their potential impact on Alzheimer's disease (AD): detection of lipopolysaccharide (LPS) in AD hippocampus. Front Cell Infect Microbiol. 2017;7:318.

16. Loffredo L, Ettorre E, Zicari AM, Inghilleri M, Nocella C, Perri L, Spalice A, Fossati C, De Lucia MC, Pigozzi F, et al. Oxidative stress and gut-derived lipopolysaccharides in neurodegenerative disease: role of NOX2. Oxidative Med Cell Longev. 2020;2020:8630275.

17. Loffredo L, Zicari AM, Perri L, Carnevale R, Nocella C, Angelico F, Del Ben M, Mosca A, Zaffina S, Panera N, et al. Does Nox2 Overactivate in children with nonalcoholic fatty liver disease? Antioxid Redox Signal. 2019;30(10):1325-30.

18. Cangemi R, Pignatelli $P$, Carnevale R, Bartimoccia S, Nocella C, Falcone M, Taliani G, Violi F. Low-grade endotoxemia, gut permeability and platelet activation in community-acquired pneumonia. J Inf Secur. 2016;73(2):10714.

19. Carnevale R, Nocella C, Petrozza V, Cammisotto V, Pacini L, Sorrentino V, Martinelli O, Irace L, Sciarretta S, Frati G, et al. Localization of lipopolysaccharide from Escherichia Coli into human atherosclerotic plaque. Sci Rep. 2018;8(1):3598.

20. Carnevale R, Silvestri R, Loffredo L, Novo M, Cammisotto V, Castellani V, Bartimoccia S, Nocella C, Violi F. Oleuropein, a component of extra virgin olive oil, lowers postprandial glycaemia in healthy subjects. Br J Clin Pharmacol. 2018;84(7):1566-74.

21. Wang X, Pinto-Duarte A, Sejnowski TJ, Behrens MM. How Nox2-containing NADPH oxidase affects cortical circuits in the NMDA receptor antagonist model of schizophrenia. Antioxid Redox Signal. 2013;18(12):1444-62.

22. Ticinesi A, Tana C, Nouvenne A, Prati B, Lauretani F, Meschi T. Gut microbiota, cognitive frailty and dementia in older individuals: a systematic review. Clin Interv Aging. 2018;13:1497-511.

23. Kowalski K, Mulak A. Brain-gut-microbiota Axis in Alzheimer's disease. J Neurogastroenterol Motil. 2019;25(1):48-60.

24. Sasmita AO. Modification of the gut microbiome to combat neurodegeneration. Rev Neurosci. 2019;30(8):795-805.

25. Qin L, Wu X, Block ML, Liu Y, Breese GR, Hong JS, Knapp DJ, Crews FT. Systemic LPS causes chronic neuroinflammation and progressive neurodegeneration. Glia. 2007;55(5):453-62.

26. Benusa SD, George NM, Sword BA, DeVries GH, Dupree JL. Acute neuroinflammation induces AIS structural plasticity in a NOX2-dependent manner. J Neuroinflammation. 2017;14(1):116.

27. Mora S, Martin-Gonzalez E, Flores P, Moreno M. Neuropsychiatric consequences of childhood group A streptococcal infection: A systematic review of preclinical models. Brain Behav Immun. 2019;(19):30214-4.

28. Fasano A, Not T, Wang W, Uzzau S, Berti I, Tommasini A, Goldblum SE. Zonulin, a newly discovered modulator of intestinal permeability, and its expression in coeliac disease. Lancet. 2000;355(9214):1518-9.
29. Sapone A, de Magistris L, Pietzak M, Clemente MG, Tripathi A, Cucca F, Lampis R, Kryszak D, Carteni M, Generoso M, et al. Zonulin upregulation is associated with increased gut permeability in subjects with type 1 diabetes and their relatives. Diabetes. 2006;55(5):1443-9.

\section{Publisher's Note}

Springer Nature remains neutral with regard to jurisdictional claims in published maps and institutional affiliations.

\section{Ready to submit your research? Choose BMC and benefit from:}

- fast, convenient online submission

- thorough peer review by experienced researchers in your field

- rapid publication on acceptance

- support for research data, including large and complex data types

- gold Open Access which fosters wider collaboration and increased citations

- maximum visibility for your research: over $100 \mathrm{M}$ website views per year

At BMC, research is always in progress.

Learn more biomedcentral.com/submissions 